\title{
Primary gastroduodenal amyloidosis
}

Amyloidosis is characterized by tissue deposition of insoluble fibrillar proteins in various organs [1]. In humans, more than 23 different and unrelated proteins are known to form amyloid fibrils [2]. Amyloidosis is divided into primary (i.e. idiopathic) and secondary amyloidosis (i.e. associated with chronic inflammatory conditions, and infectious and neoplastic disorders) [1]. Primary amyloidosis is extremely rare in the gastrointestinal tract. Fewer than $1 \%$ of patients with primary amyloidosis in the gastrointestinal tract have any symptoms [3]. We report a case of primary gastroduodenal amyloidosis in which endoscopic ultrasound (EUS) was instrumental in the work-up.

A 76-year-old man presented with a history of fatigue, dyspepsia, and anemia. An upper-gastrointestinal endoscopy revealed prominent gastric folds and gastropathy ( $\bullet$ Fig. 1a).

Gastric biopsies showed only signs of unspecific inflammation. The patient developed diarrhea and weight loss. Abdominal computed tomography (CT) showed unspecific gastric wall thickening. The initial suspicion was a malignant disease such as scirrhous carcinoma, which led to an EUS referral. EUS revealed gastric wall thickening ( Fig. 1 b) and a complete lack of normal sonographic layers in the stomach wall, suggestive of an infiltrative disease ( $\bullet$ Fig. 1b), but no sign of malignancy. Another upper-gastrointestinal endoscopy was undertaken with new biopsies from the stomach and bulbus duodeni. Congo red staining revealed amorphous eosinophilic infiltrates ( $\bullet$ Fig. 2 a) and green birefringence under polarized light ( Fig. 2b), which is diagnostic for amyloidosis [1].

It may be suggested that tissue deposition of insoluble proteins causes the sonographic disappearance of normal gastric wall layers in amyloidosis. This patient had no systemic disease such as a chronic immune disease or neoplastic disorder, which are the most common causes of secondary amyloidosis. Thus this patient had a primary amyloidosis in the gastroduodenum. In conclusion, this case highlights the use of EUS in the work-up of patients with suspected amyloidosis in the stomach and duodenum.
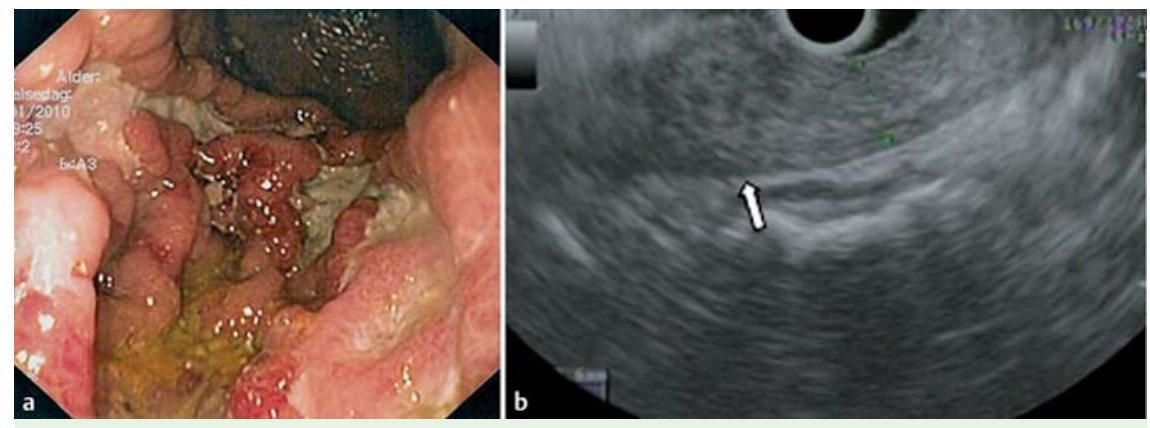

Fig. 1 a Endoscopic image of the stomach. The endoscopic view shows prominent and irregular gastric folds in the corpus, with food remnants. $\mathbf{b}$ Endoscopic ultrasound (EUS) image of the stomach, revealing increased gastric wall thickening $(9.6 \mathrm{~mm})$ and loss of the typical $1-3$ sonographic wall layers, although layer 4 (muscularis propria) is normal (arrow).

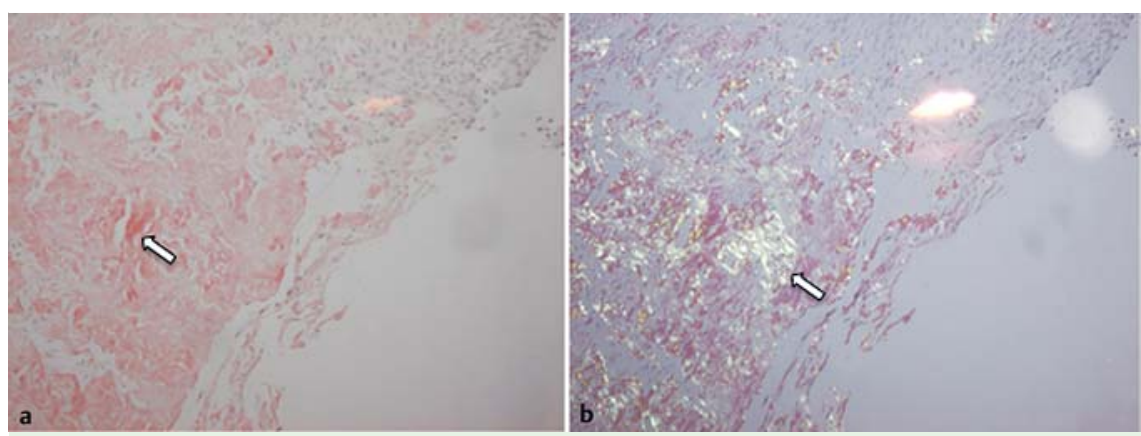

Fig. 2 Photomicrographs from gastric biopsies, stained with Congo red. a Sample viewed with normal light revealed amorphous eosinophilic infiltrates in the interstitium (arrow). b Sample viewed with polarized light showed green birefringence (arrow) of areas stained with Congo red, which is diagnostic for amyloidosis.

Endoscopy_UCTN_Code_CCL_1AB_2AD_3AD

\section{Competing interests: None}

\section{T. Grape ${ }^{1}$, G. Wurm Johansson²,}

M. Eriksson ${ }^{1}$, E. Toth ${ }^{2}$, H. Thorlacius ${ }^{2}$

1 Department of Surgery, Central Hospital, Kristianstad, Sweden

2 Department of Clinical Sciences, Lund University, Malmö, Sweden

\section{References}

1 Ebert EC, Nagar M. Gastrointestinal manifestations of amyloidosis. Am J Gastroenterol 2008; 103: 776- 787

2 Buxbaum JN. The systemic amyloidoses. Curr Opin Rheumatol 2004; 16: 67-75

3 Menke DM, Kyle RA, Fleming CR et al. Symptomatic gastric amyloidosis in patients with primary systemic amyloidosis. Mayo Clin Proc 1993; 68: 763 - 767
Bibliography

DOI $10.1055 / \mathrm{s}-0030-1256420$

Endoscopy 2011; 43: E288

(c) Georg Thieme Verlag KG Stuttgart · New York · ISSN 0013-726X

Corresponding author Henrik Thorlacius, MD

Department of Surgery and Clinical Sciences

Skåne University Hospital

Lund University

S-205 02 Malmö

Sweden

Fax: +46-40-336207

henrik.thorlacius@med.lu.se 